\title{
Cytarabine-asparagine Prodrug BST-236
}

National Cancer Institute

\section{Source}

National Cancer Institute. Cytarabine-asparagine Prodrug BST-236. NCI Thesaurus.

Code C153327.

A small molecule pro-drug consisting of cytarabine, an antimetabolite analog of cytidine with a modified arabinose sugar moiety, covalently bonded to asparagine, with potential antineoplastic activity. Upon intravenous administration, cytarabine-asparag ine prodrug BST -236 targets cancer cells, which often lack asparagine synthetase and are dependent on an external source of amino acids due to their high metabolic rate. Once the prodrug is inside target cells, the cytarabine component is cleaved and competes with cytidine for incorporation into DNA. The arabinose sugar moiety of cytarabine sterically hinders the rotation of the molecule within DNA, resulting in cell cycle arrest, specifically during the $S$ phase of replication. Cytarabine also inhibits DNA polymerase, resulting in a decrease in DNA replication and repair. Because BST-236 specifically targets cancer cells, it may spare normal tissues from cytarabine-related toxicities. 